\title{
Cut-Off Values of the Post-Intensive Care Syndrome Questionnaire for the Screening of Unplanned Hospital Readmission within One Year
}

\author{
Kang, Jiyeon ${ }^{1 \oplus} \cdot$ Jeong, Yeon Jin $^{2 \odot} \cdot$ Hong, Jiwon ${ }^{1 \oplus}$ \\ ${ }^{1}$ College of Nursing, Dong-A University, Busan \\ ${ }^{2}$ Department of Nursing, Dongju College, Busan, Korea
}

\begin{abstract}
Purpose: This study aimed to assign weights for subscales and items of the Post-Intensive Care Syndrome questionnaire and suggest optimal cut-off values for screening unplanned hospital readmissions of critical care survivors. Methods: Seventeen experts participated in an analytic hierarchy process for weight assignment. Participants for cut-off analysis were 240 survivors who had been admitted to intensive care units for more than 48 hours in three cities in Korea. We assessed participants using the 18-item Post-Intensive Care Syndrome questionnaire, generated receiver operating characteristic curves, and analysed cut-off values for unplanned readmission based on sensitivity, specificity, and positive likelihood ratios. Results: Cognitive, physical, and mental subscale weights were $1.13,0.95$, and 0.92 , respectively. Incidence of unplanned readmission was 25.4\%. Optimal cut-off values were 23.00 for raw scores and 23.73 for weighted scores (total score 54.00), with an area of under the curve (AUC) of .933 and .929, respectively. There was no significant difference in accuracy for original and weighted scores. Conclusion: The optimal cut-off value accuracy is excellent for screening of unplanned readmissions. We recommend that nurses use the Post-Intensive Care Syndrome Questionnaire to screen for readmission risk or evaluating relevant interventions for critical care survivors.
\end{abstract}

Key words: Intensive Care Unit; Patient Readmission; Reproducibility of Results; ROC Curve; Survivor

\section{INTRODUCTION}

The focus of critical care outcomes has shifted from survival to long-term functional status and quality of life [1]. Although individual recovery trajectories for critical illness vary, intensive care unit (ICU) survivors often do not return to their previous state for a long period post-discharge [2]. This gap can be described as Post-Intensive Care Syndrome (PICS), referring to cognitive, physical, or mental health problems that develop or worsen related to ICU treatment [3]. Over 50\% of ICU survivors experience varying degrees of PICS, and PICS is known to last for many years [4].
As PICS received more research attention, another issue appeared, in that the measures for PICS evaluation were too diverse [5,6]. A scoping review of 425 studies on ICU survivorship reported that approximately 250 different tools were used to measure post-ICU outcomes [7]. Excessive diversity of tools to measure a variable can hinder the integration of study findings; therefore, standardization of PICS measurement is necessary. Needham et al. [8] proposed a core outcome set using the Delphi technique for assessing PICS in acute respiratory failure survivors. This set comprises existing tools for evaluating physical, mental, and cognitive functions such as the Hospital Anxiety and Depression Scale (HADS), Impact of Event Scale-Revised

* This work was presented at 2020 Korean Society of Nursing Science Conference, October, 2020, Seoul, South Korea.

Address reprint requests to: Hong, Jiwon

College of Nursing, Dong-A University, 32 Daesingongwon-ro, Seo-gu, Busan 49201, Korea

Tel: +82-51-240-2871 Fax: +51-240-2920Ｅ-mail: superj419@naver.com

Received: September 24, 2020 Revised: November 20, 2020 Accepted: November 30, 2020 Published online December 31, 2020

This is an Open Access article distributed under the terms of the Creative Commons Attribution NoDerivs License. (http://creativecommons. org/licenses/by-nd/4. 0)

If the original work is properly cited and retained without any modification or reproduction, it can be used and re-distributed in any format and medium. 
(IES-R), Montreal Cognitive Assessment (MoCA), 36-Item Short Form Survey (SF-36), and EuroQol-5 Dimension (EQ-5D). However, the types of tools in the core set differ, and the total item number is still large. In addition, even though they were not developed for critically ill patients, they are commonly used in critical care research. This may lead to measurement validity or sensitivity issues.

Contrastingly, a new PICS questionnaire (PICSq) was developed through in-depth interviews and surveys with 536 ICU survivors, and appropriate reliability and validity were reported [9]. Because this tool was developed based on the experience of ICU survivors, it may be more appropriate to assess PICS than other tools. Additionally, the scope of application is wider, because it is targeted at the general ICU population, rather than a specific group of patients. Clinicians can use PICSq scores to screen for PICS in ICU survivors, evaluate intervention effectiveness, or track long-term outcomes [9]. However, PICSq cut-off values for ICU survivor prognosis such as unplanned hospital readmissions have not been investigated. Thus, defined optimal cut-off values will further improve the applicability and usability of the PICSq.

ICU survivorship is associated with significant hospital resource utilization and costs $[10,11]$. According to a United Kingdom national data analysis, $81.7 \%$ of survivors experienced more than one hospital readmission within five years, of which $54 \%$ were unplanned [11]. Unplanned hospital readmissions are an indicator of cost and quality improvements, placing strain on families, society, and individual survivors [12]. Internationally, ICU survivors spend 18,847 to 148,454 US dollars annually on medical expenses [13]. The complex health and psychosocial issues of ICU survivors, namely, PICS, contribute to early readmission after discharge [14]. Experts also noted that early intervention for PICS is important to lower unplanned hospital readmissions [15]. Providing optimal cut-off values for the PICSq for screening patients at high risk of unplanned hospital readmission may help initiate early intervention.

Additionally, since the PICSq is a newly developed tool, expert consensus is needed on whether all items have the same weight. Weights can be imposed through a subjective method of assessing the importance of each item or through a statistical analysis of responses [16]. The analytic hierarchy process (AHP) is a method of mathematically integrating subjective weights. It allows experts to assign relative weights to each item by repeated ranking and comparisons between pairs of items in a tool based on their experience [17]. The AHP compensates for the risk that statistical analysis results may not be consistent with the weights in the real world [18]. Therefore, it is an appropriate method for evaluating the weight of the PICSq, which is still lacking in clinical and research applications. This study aimed to (1) assign AHP weights for the PICSq items and subscales, and (2) suggest optimal cut-off values for raw and weighted PICSq scores for the screening of the unplanned readmission of ICU survivors.

\section{METHODS}

\section{Study design}

This was a methodological study with a cross-sectional design to suggest PICSq cut-off values for screening unplanned readmissions of survivors after ICU discharge.

\section{Participants}

Participants were survivors who had been discharged home after ICU treatment. Inclusion criteria were $\geq 18$ years of age, ICU stay $\geq 48$ hours, and $<1$ year post-discharge. Exclusion criteria were cognitive impairment before ICU treatment and communication difficulties for the survey. We also excluded survivors who were discharged to rehabilitation or long-term care facilities, because the definition of readmission could be different.

We calculated the sample size in two ways. First, the sample size for PICS comparison between groups was calculated using G*Power 3.1.9.7. The minimum number of participants required for an ANOVA was 231 when the number of groups (categories of variables) was 7 , effect size was .25 (medium), significance level was .05 , and power was .80 . Second, the minimum sample size for ROC curve analysis was 62 (95\% confidence interval, power of the study $80 \%$ with $\alpha$ error $5 \%$, maximum prediction .70) calculated using MedCalc 19.2.0 statistical software. We adopted the larger of the two sample sizes. We distributed questionnaires to 255 survivors, considering a dropout rate of about $10 \%$, of which 240 were used for final analysis, excluding 15 with missing responses. 


\section{Measurements}

The PICSq, developed by Jeong and Kang [9], is a self-report measure comprising 18 items and three subscales that assess cognitive, physical, and mental impairments. Items are rated using a four-point Likert scale ( 0 = "never," 1 = "sometimes," $2=$ "most often," 3 = "always"). Total scores range between 0 and 54, and subscale scores range between 0 and 18. Higher scores indicate more severe PICS. At the time of development, reliability of the PICSq was Cronbach's $\alpha=.94$. In the present study Cronbach's $\alpha=.92$.

Weighted PICS (wPICS) was calculated through the AHP, a process of classifying items into criteria and alternatives, and calculating the importance by comparing them in pairs [18]. In this study, we layered the AHP structure by substituting subscales and items of the PICSq. The standard of sample size required for the AHP is not well known, but the larger the sample, the more difficult it is to ensure the consistency of response [17,19]. Based on a systematic review that 10 20 experts participated in most AHP studies [17], we recruited 17 critical care or rehabilitation experts, including 11 ICU nurses, an intensivist, two rehabilitation nurses, and three rehabilitation physicians. They assessed relative importance through a pairwise comparison of the PICSq's subscales (cognitive, physical, and mental) and items using a 9-point scale. We calculated the consistency ratio (CR) to evaluate the consistency between each pairwise comparison. If the consistency is perfect, $\mathrm{CR}=0$, and usually $\mathrm{CR}<.2$ is an acceptable level $[17,20,21]$. In this study, the criteria for consistency were satisfied with $\mathrm{CR}=.05$ to .16 for each item. We input the responses of 17 experts into Expert Choice software (Expert Choice Riskion ${ }^{\circledR}$, Arlington, Virginia) to calculate the weights of the three subscales and 18 items of the PICSq.

Additionally, hospital readmission was one of the survey items, and each participant responded directly. It was explained to the participants that planned readmission (i.e., for chemotherapy or rehabilitation) should be excluded. They were asked to respond only with regard to unplanned readmissions after hospital discharge.

\section{Procedure}

We conducted a survey from November 19, 2018 to June 28,
2019. Participants were publicly recruited by posting notices on bulletin boards in outpatient clinics at four tertiary hospitals located in three cities in Korea. When an ICU survivor indicated willingness to participate, research assistants reviewed whether the person met selection criteria. Selected participants completed the survey in designated meeting rooms, which took about five minutes.

\section{Ethical considerations}

The Dong-A University Institutional Review Board approved this study (2-1040709-AB-N-01-201810-HR-031-02, 2-10407 09-AB-N-01-201810-HR-031-03). All participants provided written consent after being informed of the study's purpose and methods.

\section{Statistical analysis}

Data were analysed using IBM SPSS 25.0 (IBM Corp., Armonk, NY) and MedCalc 19.2.0 (MedCalc Software, Mariakerke, Belgium),

Participant demographics and ICU characteristics were analysed by calculating descriptive statistics such as frequency, percentage, mean, and standard deviation. PICSq mean differences according to participant characteristics were analysed by independent sample t-test and ANOVA. The scheffe test was used a post-hoc test. Differences in participant characteristics depending on whether or not there was an unplanned readmission were analysed by the chi-square test and independent sample t-test.

We generated Receiver Operating Characteristics (ROC) curves to identify changes in sensitivity and specificity at each cut-off value for unplanned readmission. Optimal cut-off values for PICSq and wPICS scores were presented considering positive likelihood ratio (LR) as well as sensitivity and specificity. In general, a value with high sensitivity and specificity is selected as the cut-off score [22]. However, there is a limitation that sensitivity and specificity are affected by the prevalence. In order to compensate for this, the positive likelihood ratio, which is not related to the prevalence, should also be considered when determining the cut-off score [23]. Based on the opinions that a LR of less than 3 is not useful for clinical decisions [24,25], we selected scores with a LR of 3 or more while having both high sensitivity and specificity as the optimal cut-off values. Accuracy differences between PICSq and wPICS cut-off values were analysed 
by the $z$-test for area under the ROC curve (AUC).

\section{RESULTS}

\section{Weights of subscales and items}

Table 1 shows the weights of the three subscales and 18 items of the questionnaire calculated via AHP. The expert group weighted the highest on the cognitive subscale and lowest on the mental subscale. On the cognitive subscale, the item with the highest weight was "I am confused with date or time," and the lowest item was "It's hard to memorize numbers." The item with the highest weight on the physical subscale was "I get tired easily, and the item with the lowest weight was "My sexual performance has deteriorated." For the mental subscale, "I have no hope" had the highest weight, and "I am easily startled" had the lowest weight.

The formula for calculating $w$ PICS was as follows:

$w \mathrm{PICS}_{\text {total }}=1.13 * w \mathrm{PICS}_{\text {cognitive }}+0.95 * w \mathrm{PICS}_{\text {physical }}+0.92 * w \mathrm{PICS}_{\text {mental }}$ ${ }_{w P I C S}$ cognitive $=0.53 *$ Item $1+0.92 *$ Item $2+1.10 *$ Item $3+0.99 *$ Item $4+0.69 *$ Item $5+1.77 *$ Item 6 ${ }_{w P I C S}$ physical $=0.63 *$ Item $7+1.05 *$ Item $8+0.85 *$ Item $9+0.36 *$ Item $10+1.65 *$ Item $11+1.46 *$ Item 12 $w \mathrm{PICS}_{\text {mental }}=0.90 *$ Item $13+0.80 *$ Item $14+1.16 *$ Item $15+1.23 *$ Item $16+0.66 *$ Item $17+1.25 *$ Item 18

\section{Participant characteristics and post-intensive care syndrome}

Of the 240 participants, the mean age was $61.4 \pm 14.54$ years, and $70.8 \%$ were male. The majority $(86.3 \%)$ of primary caregivers were families. Before ICU admission, $45.0 \%$ of the participants were unemployed; however, $70.0 \%$ were unemployed post-discharge. Monthly household income of most participants (79.6\%) was less than 3,000 US dollars.

Participants' diagnostic categories were cardiovascular (25.4\%), neurologic (21.7\%), and gastrointestinal diseases (12.1\%). They were admitted to surgical (35.8\%), neurologic (23.8\%), and medical ICUs (16.3\%). More than half (57.9\%) had surgery, and $67.9 \%$ were admitted via emergency rooms. About half (55.8\%) of ICU admissions were planned. Among the participants, 5.8\% received $\mathrm{CPR}$ and $31.3 \%$ were on mechanical ventilation. The mean number of ICU days was $8.38 \pm 9.19$, and mean number of hospital admission days was $36.10 \pm 31.16$. The mean number of months post-discharge was $6.82 \pm 4.06$. About one-fourth (25.8\%) had experienced unplanned readmissions (Table 2).

The mean PICSq raw score was $18.13 \pm 11.84$. Mean PICSq scores were significantly higher in the following groups: $\geq 60$ years old $(t=-1.98, p=.049)$, professional caregiver service $(\mathrm{F}=6.58, p=.002)$, previous unemployment $(\mathrm{t}=-3.32$,

Table 1. Weights of Post Intensive Care Syndrome Questionnaire Subscales and Items

\begin{tabular}{|c|c|c|c|c|c|}
\hline Subscales & Weights & Rank & Items & Weights & Rank \\
\hline \multirow[t]{6}{*}{ Cognitive impairment } & 1.13 & 1 & 1. It is hard to memorize numbers. & 0.53 & 6 \\
\hline & & & 2. People around me say that I repeat what I said before. & 0.92 & 4 \\
\hline & & & 3. It is hard for me to find the way. & 1.10 & 2 \\
\hline & & & 4. I cannot concentrate on reading. & 0.99 & 3 \\
\hline & & & 5. Money management is difficult. & 0.69 & 5 \\
\hline & & & 6. I am confused with date or time. & 1.77 & 1 \\
\hline \multirow[t]{6}{*}{ Physical impairment } & 0.95 & 2 & 7. My joints are stiff. & 0.63 & 5 \\
\hline & & & 8. My handgrip is weak. & 1.05 & 3 \\
\hline & & & 9. I can hardly climb the stairs. & 0.85 & 4 \\
\hline & & & 10. My sexual performance has deteriorated. & 0.36 & 6 \\
\hline & & & 11. I get tired easily. & 1.65 & 1 \\
\hline & & & 12. I feel sick everywhere in my body. & 1.46 & 2 \\
\hline \multirow[t]{6}{*}{ Mental impairment } & 0.92 & 3 & 13. My heart is stuffy. & 0.90 & 4 \\
\hline & & & 14. I have nightmares. & 0.80 & 5 \\
\hline & & & 15. I am worried. & 1.16 & 3 \\
\hline & & & 16. I am annoyed or angry. & 1.23 & 2 \\
\hline & & & 17. I am easily startled. & 0.66 & 6 \\
\hline & & & 18. I have no hope. & 1.25 & 1 \\
\hline
\end{tabular}


Table 2. Participants' Characteristics and Post-Intensive Care Syndrome

$(N=240)$

\begin{tabular}{|c|c|c|c|c|c|c|}
\hline Variables & Categories & $\begin{array}{l}\mathrm{PICS} \\
\mathrm{M} \pm \mathrm{SD}\end{array}$ & $\operatorname{tor} F(p)$ & $\begin{array}{l}w P I C S \\
M \pm S D\end{array}$ & $\operatorname{tor} F(p)$ & Scheffe test \\
\hline \multirow[t]{2}{*}{ Gender } & Man & $17.90 \pm 11.96$ & $-0.46(.647)$ & $18.17 \pm 12.08$ & $-0.67(.507)$ & \\
\hline & Woman & $18.97 \pm 11.60$ & & $19.30 \pm 11.80$ & & \\
\hline \multirow[t]{2}{*}{ Age (yr) } & $<60$ & $16.30 \pm 10.89$ & $-1.98(.049)$ & $16.52 \pm 10.87$ & $-2.12(.035)$ & \\
\hline & $\geq 60$ & $19.36 \pm 12.32$ & & $19.84 \pm 12.55$ & & \\
\hline \multirow[t]{3}{*}{ Primary caregiver } & Familya & $17.16 \pm 11.69$ & $6.58(.002)$ & $17.58 \pm 11.86$ & $5.96(.003)$ & $b>a$ \\
\hline & Professional caregiver ${ }^{b}$ & $28.58 \pm 12.98$ & & $28.74 \pm 13.41$ & & \\
\hline & None & $21.62 \pm 9.31$ & & $21.68 \pm 9.41$ & & \\
\hline \multirow[t]{2}{*}{ Job prior to ICU admission } & Yes & $15.88 \pm 11.37$ & $-3.32(.001)$ & $16.15 \pm 11.49$ & $-3.43(.001)$ & \\
\hline & No & $20.87 \pm 11.86$ & & $21.67 \pm 12.02$ & & \\
\hline \multirow[t]{2}{*}{ Current job } & Yes & $11.76 \pm 10.00$ & $-5.81(<.001)$ & $12.05 \pm 10.13$ & $-5.81(<.001)$ & \\
\hline & No & $20.85 \pm 11.54$ & & $21.26 \pm 11.68$ & & \\
\hline \multirow[t]{2}{*}{ Changes in work intensity } & Same as before & $10.13 \pm 9.78$ & $-2.49(.015)$ & $10.36 \pm 10.02$ & $-2.56(.013)$ & \\
\hline & Easier work & $16.67 \pm 9.23$ & & $17.14 \pm 8.89$ & & \\
\hline \multirow{5}{*}{$\begin{array}{l}\text { Monthly income (US } \\
\text { dollars) }\end{array}$} & None & $18.63 \pm 12.10$ & $0.78(.539)$ & $18.76 \pm 12.35$ & $0.74(.568)$ & \\
\hline & $<1,000$ & $18.35 \pm 11.88$ & & $18.71 \pm 12.10$ & & \\
\hline & $1,000 \sim<2,000$ & $19.79 \pm 9.21$ & & $20.49 \pm 9.39$ & & \\
\hline & $2,000 \sim<3,000$ & $15.03 \pm 12.00$ & & $15.59 \pm 12.07$ & & \\
\hline & $\geq 3,000$ & $18.14 \pm 12.88$ & & $18.55 \pm 12.90$ & & \\
\hline \multirow[t]{7}{*}{ Diagnosis } & Cardiac disease & $20.67 \pm 12.18$ & $1.84(.093)$ & $21.01 \pm 12.14$ & $1.67(.130)$ & \\
\hline & Neurologic disease & $13.94 \pm 11.68$ & & $14.39 \pm 12.19$ & & \\
\hline & Trauma disease & $18.30 \pm 10.95$ & & $18.47 \pm 11.14$ & & \\
\hline & Gastrointestinal disease & $17.55 \pm 10.69$ & & $18.26 \pm 10.67$ & & \\
\hline & Respiratory disease & $20.64 \pm 12.90$ & & $20.74 \pm 13.12$ & & \\
\hline & Renal disease & $18.89 \pm 9.80$ & & $19.05 \pm 9.72$ & & \\
\hline & Others & $17.57 \pm 12.67$ & & $18.23 \pm 12.99$ & & \\
\hline \multirow[t]{5}{*}{ Type of ICU } & Surgicala & $19.53 \pm 12.77$ & $5.87(<.001)$ & $20.17 \pm 13.01$ & $6.10(<.001)$ & $a, d>c$ \\
\hline & Neurological ${ }^{b}$ & $18.70 \pm 11.75$ & & $18.95 \pm 11.74$ & & \\
\hline & Cardiovascularc & $12.15 \pm 10.41$ & & $12.29 \pm 10.57$ & & \\
\hline & Medical $^{d}$ & $21.26 \pm 8.51$ & & $21.53 \pm 8.60$ & & \\
\hline & Others ${ }^{\mathrm{e}}$ & $29.75 \pm 10.87$ & & $30.14 \pm 9.69$ & & \\
\hline \multirow[t]{2}{*}{ Surgery } & Yes & $19.08 \pm 11.77$ & $1.47(.143)$ & $19.44 \pm 11.92$ & $1.43(.153)$ & \\
\hline & No & $16.81 \pm 11.86$ & & $17.20 \pm 12.02$ & & \\
\hline \multirow[t]{2}{*}{ Route of admission to ICU } & ED & $19.00 \pm 12.16$ & $1.67(.096)$ & $19.33 \pm 12.30$ & $1.58(.115)$ & \\
\hline & Others & $16.27 \pm 10.97$ & & $16.72 \pm 11.18$ & & \\
\hline \multirow[t]{2}{*}{ ICU admission } & Planned & $14.98 \pm 10.76$ & $-4.85(<.001)$ & $15.34 \pm 10.94$ & $-4.80(<.001)$ & \\
\hline & Unplanned & $22.10 \pm 11.99$ & & $22.49 \pm 12.11$ & & \\
\hline \multirow{2}{*}{$\begin{array}{l}\text { Cardiopulmonary } \\
\text { resuscitation }\end{array}$} & Yes & $17.93 \pm 10.89$ & $-0.06(.949)$ & $18.02 \pm 11.15$ & $-0.15(.879)$ & \\
\hline & No & $18.14 \pm 11.91$ & & $18.53 \pm 12.06$ & & \\
\hline \multirow[t]{2}{*}{ Mechanical ventilation } & Yes & $20.87 \pm 11.44$ & $2.45(.015)$ & $21.36 \pm 11.55$ & $2.52(.012)$ & \\
\hline & No & $16.88 \pm 11.84$ & & $17.19 \pm 11.99$ & & \\
\hline \multirow[t]{3}{*}{ ICU admission days } & $2 \sim<7^{a}$ & $14.04 \pm 10.13$ & $23.23(<.001)$ & $14.37 \pm 10.32$ & $23.18(<.001)$ & $b, c>a$ \\
\hline & $7 \sim 13^{b}$ & $21.65 \pm 11.76$ & & $21.98 \pm 11.85$ & & \\
\hline & $\geq 14^{c}$ & $25.70 \pm 11.73$ & & $26.24 \pm 11.81$ & & \\
\hline \multirow[t]{2}{*}{ Hospital admission days } & $<14$ & $8.87 \pm 8.16$ & $-8.69(<.001)$ & $8.85 \pm 8.19$ & $-9.02(<.001)$ & \\
\hline & $\geq 14$ & $20.88 \pm 11.37$ & & $21.36 \pm 11.44$ & & \\
\hline \multirow[t]{2}{*}{ Months after discharge } & $<6$ & $15.76 \pm 11.44$ & $-2.58(.011)$ & $16.18 \pm 11.48$ & $-2.49(.013)$ & \\
\hline & $\geq 6$ & $19.73 \pm 11.87$ & & $20.07 \pm 12.11$ & & \\
\hline \multirow[t]{2}{*}{ Unplanned readmission } & Yes & $31.34 \pm 7.36$ & $15.23(<.001)$ & $31.83 \pm 7.46$ & $15.14(<.001)$ & \\
\hline & No & $13.52 \pm 9.38$ & & $13.85 \pm 9.54$ & & \\
\hline
\end{tabular}

$\mathrm{ED}=$ Emergency department; ICU = Intensive care unit; $\mathrm{M}=$ Mean; PICS = Post-intensive care syndrome; wPICS = weighted PICS; SD = Standard deviation. 
Table 3. Participants' Characteristics by Unplanned Hospital Readmission

$(N=240)$

\begin{tabular}{|c|c|c|c|c|c|c|}
\hline \multirow[t]{2}{*}{ Variables } & \multirow[t]{2}{*}{ Categories } & \multirow[t]{2}{*}{$\mathrm{n}(\%)$} & \multirow{2}{*}{$\begin{array}{c}\text { Readmission } \\
\text { group }(n=62) \\
n(\%) \text { or } M \pm S D\end{array}$} & \multirow{2}{*}{$\begin{array}{l}\begin{array}{c}\text { Non-readmission } \\
\text { group }(n=178) \\
n(\%) \text { or } M \pm S D\end{array}\end{array}$} & \multirow[t]{2}{*}{ tor $\chi^{2}$} & \multirow[t]{2}{*}{$p$} \\
\hline & & & & & & \\
\hline \multirow[t]{2}{*}{ Gender } & Man & $170(70.8)$ & $43(69.4)$ & $127(71.3)$ & 0.09 & .766 \\
\hline & Woman & $70(29.2)$ & $19(30.6)$ & $51(28.7)$ & & \\
\hline Age (yr) & & & $64.5 \pm 13.85$ & $60.3 \pm 14.65$ & 2.00 & .047 \\
\hline \multirow{3}{*}{ Primary caregiver } & Family & $207(86.3)$ & $49(79.0)$ & $158(88.8)$ & 6.55 & .030 \\
\hline & Professional caregiver & $12(5.0)$ & $7(11.3)$ & $5(2.8)$ & & \\
\hline & None & $21(8.7)$ & $6(9.7)$ & $15(8.4)$ & & \\
\hline \multirow[t]{2}{*}{ Job prior to ICU admission } & Yes & $132(55.0)$ & $24(38.7)$ & $108(60.7)$ & 8.96 & .003 \\
\hline & No & $108(45.0)$ & $38(61.3)$ & 70 (39.3) & & \\
\hline \multirow[t]{2}{*}{ Current job } & Yes & $72(30.0)$ & $6(9.7)$ & $66(37.1)$ & 16.44 & $<.001$ \\
\hline & No & $168(70.0)$ & $56(90.3)$ & $112(62.9)$ & & \\
\hline \multirow[t]{2}{*}{ Changes in work intensity } & Same as before & $54(75.0)$ & $2(33.3)$ & $52(78.8)$ & 6.06 & .031 \\
\hline & Easier work & $18(25.0)$ & $4(66.7)$ & $14(21.2)$ & & \\
\hline \multirow[t]{5}{*}{ Monthly income (US dollars) } & None & $76(31.7)$ & $16(25.8)$ & $60(33.7)$ & 1.95 & .745 \\
\hline & $<1,000$ & $48(20.0)$ & $15(24.2)$ & $33(18.5)$ & & \\
\hline & $1,000 \sim<2,000$ & $33(13.7)$ & $9(14.6)$ & $24(13.5)$ & & \\
\hline & $2,000 \sim<3,000$ & $34(14.2)$ & $10(16.1)$ & $24(13.5)$ & & \\
\hline & $\geq 3,000$ & $49(20.4)$ & $12(19.3)$ & $37(20.8)$ & & \\
\hline \multirow[t]{7}{*}{ Diagnosis } & Cardiac disease & $61(25.4)$ & $21(33.9)$ & $40(22.5)$ & 8.83 & .178 \\
\hline & Neurologic disease & $52(21.7)$ & $7(11.3)$ & $45(25.3)$ & & \\
\hline & Trauma disease & $30(12.5)$ & $7(11.3)$ & $23(12.9)$ & & \\
\hline & Gastrointestinal disease & $29(12.1)$ & $7(11.3)$ & $22(12.4)$ & & \\
\hline & Respiratory disease & $28(11.7)$ & $8(12.9)$ & $20(11.2)$ & & \\
\hline & Renal disease & $19(7.9)$ & $4(6.4)$ & $15(8.4)$ & & \\
\hline & Others & $21(8.7)$ & $8(12.9)$ & $13(7.3)$ & & \\
\hline \multirow[t]{5}{*}{ Type of ICU } & Surgical & $86(35.8)$ & $31(50.0)$ & $55(30.9)$ & 33.48 & $<.001$ \\
\hline & Neurological & $57(23.8)$ & $13(21.0)$ & $44(24.7)$ & & \\
\hline & Cardiovascular & $54(22.5)$ & $1(1.6)$ & $53(29.8)$ & & \\
\hline & Medical & $39(16.3)$ & $14(22.6)$ & $25(14.0)$ & & \\
\hline & Others & $4(1.6)$ & $3(4.8)$ & $1(0.6)$ & & \\
\hline \multirow[t]{2}{*}{ Surgery } & Yes & $139(57.9)$ & $40(64.5)$ & $99(55.6)$ & 1.49 & .222 \\
\hline & No & $101(42.1)$ & $22(35.5)$ & $79(44.4)$ & & \\
\hline \multirow{2}{*}{ Route of admission to ICU } & ED & $163(67.9)$ & $44(71.0)$ & $119(66.9)$ & 0.36 & .550 \\
\hline & Others & $77(32.1)$ & $18(29.0)$ & $59(33.1)$ & & \\
\hline \multirow{2}{*}{ Planned ICU admission } & Yes & $134(55.8)$ & $22(35.5)$ & $112(62.9)$ & 14.04 & $<.001$ \\
\hline & No & $106(44.2)$ & $40(64.5)$ & $66(37.1)$ & & \\
\hline \multirow[t]{2}{*}{ Cardiopulmonary resuscitation } & Yes & $14(5.8)$ & $3(4.8)$ & $11(6.2)$ & 0.15 & .698 \\
\hline & No & $226(94.2)$ & $59(95.2)$ & $167(93.8)$ & & \\
\hline \multirow[t]{2}{*}{ Mechanical ventilation } & Yes & $75(31.3)$ & $27(43.5)$ & $48(27.0)$ & 5.89 & .015 \\
\hline & No & $165(68.7)$ & $35(56.5)$ & $130(73.0)$ & & \\
\hline ICU admission days & & & $11.65 \pm 8.72$ & $7.24 \pm 9.10$ & 3.39 & .001 \\
\hline Hospital admission days & & & $52.82 \pm 32.04$ & $30.28 \pm 28.73$ & 4.90 & $<.001$ \\
\hline Months after discharge & & & $7.90 \pm 3.80$ & $6.44 \pm 4.10$ & 2.46 & .015 \\
\hline PICS & Total & & $31.34 \pm 7.36$ & $13.52 \pm 9.38$ & 15.23 & $<.001$ \\
\hline & Cognitive & & $10.03 \pm 3.55$ & $3.38 \pm 3.46$ & 12.95 & $<.001$ \\
\hline & Physical & & $11.10 \pm 2.83$ & $5.78 \pm 3.81$ & 11.57 & $<.001$ \\
\hline & Mental & & $10.21 \pm 2.61$ & $4.37 \pm 3.83$ & 13.32 & $<.001$ \\
\hline PICS, weighted & Total & & $31.83 \pm 7.46$ & $13.85 \pm 9.54$ & 15.14 & $<.001$ \\
\hline & Cognitive & & $10.16 \pm 3.49$ & $3.45 \pm 3.49$ & 10.92 & $<.001$ \\
\hline & Physical & & $11.31 \pm 2.94$ & $6.14 \pm 3.90$ & 13.53 & $<.001$ \\
\hline & Mental & & $10.43 \pm 2.58$ & $4.48 \pm 3.93$ & 15.14 & $<.001$ \\
\hline
\end{tabular}

$\mathrm{ED}=$ Emergency department; ICU = Intensive care unit; $\mathrm{M}=$ Mean; $\mathrm{PCS}=$ Post-intensive care syndrome; SD = Standard deviation.

${ }^{\dagger}$ Fisher exact test 
$p=.001)$, current unemployment $(\mathrm{t}=-5.81, p<.001)$, changes in workload $(t=-2.49, p=.015)$, admitted to medical or surgical ICU ( $\mathrm{F}=5.87, p<.001)$, unplanned ICU admission $(\mathrm{t}=-4.85$, $p<.001)$, mechanical ventilation $(\mathrm{t}=2.45, p=.015), \geq 14 \mathrm{ICU}$ days $(\mathrm{F}=23.23, p<.001), \geq 14$ hospital days $(\mathrm{t}=-8.69$, $p<.001), \geq 6$ months post-discharge $(t=-2.58, p=.011)$, and unplanned readmission $(t=15.23, p<.001)$.

Mean $w$ PICS score was $18.50 \pm 12.00$, and the differences in mean wPICS scores according to participant characteristics were similar to the raw score analysis (Table 2).

\section{Optimal cut-off values for unplanned hospital readmission}

Unplanned hospital readmission incidence within a year was 25.8\%; Table 3 shows differences in participant characteristics according to unplanned readmission. Raw and weighted total and subscale scores for the PICSq in the readmission group were significantly higher than those of the non-readmission group.

Figure 1 and Table 4 show the cut-off values of PICSq raw and weighted scores to screen the unplanned readmission of ICU survivors. Optimal cut-off values, considering sensitivity, speci-

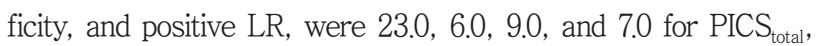

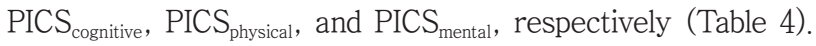
Sensitivity, specificity, positive LR, and AUC at the cut-off value of 23.0 for $\mathrm{PICS}_{\text {total }}$ were $85.5 \%, 81.5 \%, 4.61$, and .933, respectively. Additionally, we suggested optimal cut-off values of 23.73 for $w \mathrm{PICS}_{\text {total }}, 6.50$ for $w \mathrm{PICS}_{\text {connitive }}, 9.27$ for $w \mathrm{PICS}_{\text {physical }}$, and 8.42 for ${ } \mathrm{PICS}_{\text {mental }}$. The sensitivity, specificity, positive LR, and AUC for the cut-off value of $23.73 \mathrm{wPICS}_{\text {total }}$ were $85.5 \%, 80.9 \%, 4.48$, and .929 , respectively. There was no statistically significant dif-
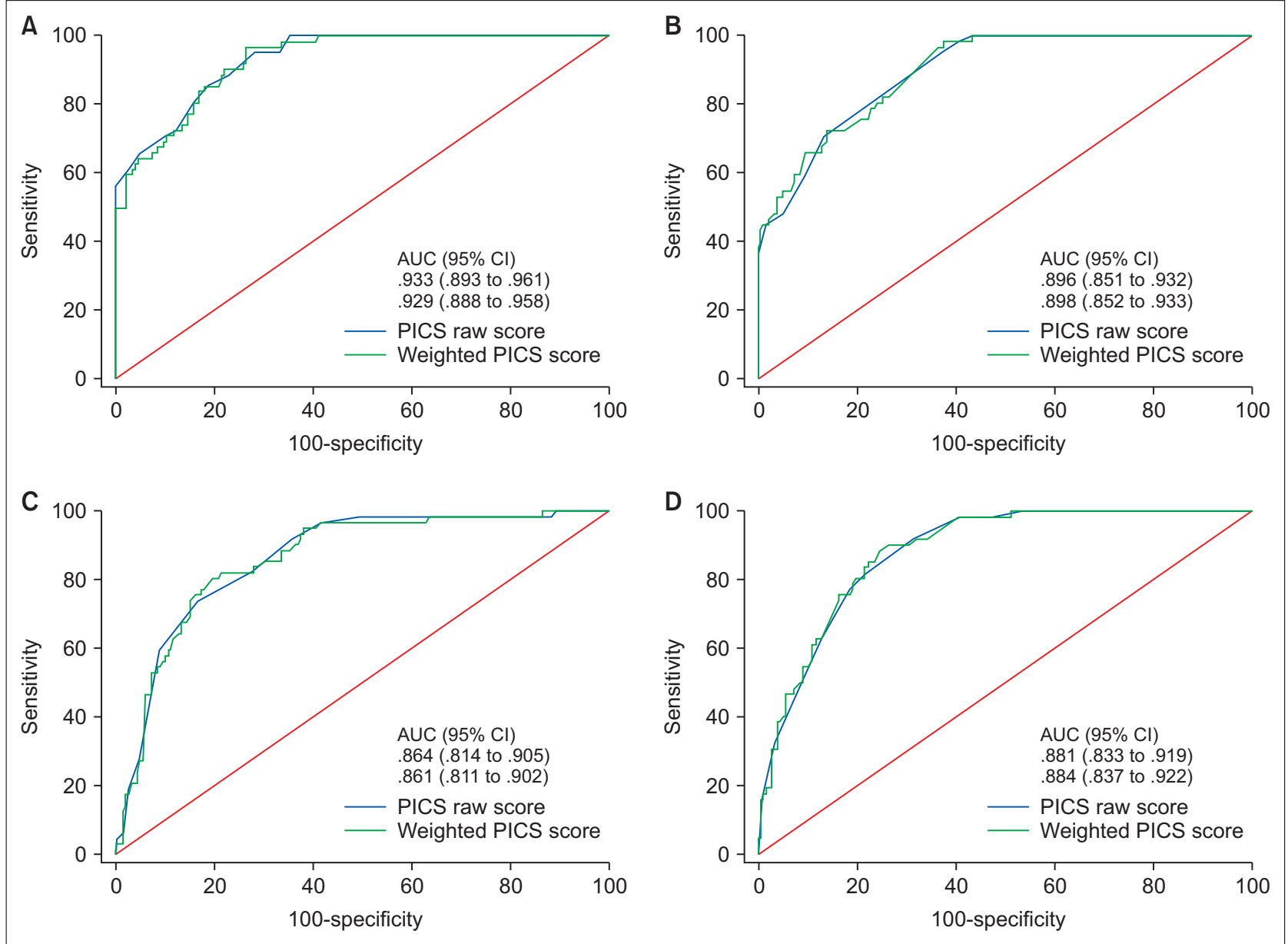

AUC = Area under the curve; $\mathrm{Cl}=$ Confidence interval; PICS = Post-intensive care syndrome; $w$ PICS = weighted PICS .

Figure 1. AUC comparison of PICS raw and weighted scores (A: PICS total, B: PICS cognitive, C: PICS physical, D: PICS mental). 
Table 4. Cut-Off Values for Unplanned Hospital Readmission ( $N=$ 240)

\begin{tabular}{|c|c|c|c|c|}
\hline Variables & $\begin{array}{l}\text { Cut-off } \\
\text { value }\end{array}$ & $\begin{array}{c}\text { Sensitivity } \\
(\%)\end{array}$ & $\begin{array}{c}\text { Specificity } \\
(\%)\end{array}$ & LR $(95 \% \mathrm{Cl})$ \\
\hline \multirow[t]{3}{*}{ PICS $_{\text {total }}$} & 22.00 & 88.7 & 77.0 & 3.85 (2.90 to 5.10$)$ \\
\hline & 23.00 & 85.5 & 81.5 & 4.61 (3.30 to 6.40$)$ \\
\hline & 24.00 & 80.7 & 84.3 & 5.13 (3.60 to 7.40$)$ \\
\hline \multirow[t]{3}{*}{$w P I C S_{\text {total }}$} & 22.88 & 90.3 & 78.1 & 4.12 (3.10 to 5.50$)$ \\
\hline & 23.73 & 85.5 & 80.9 & 4.48 (3.30 to 6.20$)$ \\
\hline & 25.76 & 74.2 & 87.6 & 6.00 (4.00 to 9.10$)$ \\
\hline \multirow{3}{*}{ PICS cognitive } & 5.00 & 95.2 & 62.9 & 2.57 (2.10 to 3.10$)$ \\
\hline & 6.00 & 80.7 & 77.0 & 3.50 (2.60 to 4.70$)$ \\
\hline & 7.00 & 71.0 & 86.5 & 5.26 (3.50 to 7.90$)$ \\
\hline \multirow[t]{3}{*}{$W P I C S_{\text {cognitive }}$} & 5.51 & 98.4 & 62.4 & 2.61 (2.20 to 3.20$)$ \\
\hline & 6.50 & 80.7 & 75.8 & 3.34 (2.50 to 4.50$)$ \\
\hline & 7.49 & 72.6 & 86.0 & 5.17 (3.50 to 7.70$)$ \\
\hline \multirow[t]{3}{*}{ PICS ${ }_{\text {physical }}$} & 8.00 & 82.3 & 72.5 & 2.99 (2.30 to 3.90$)$ \\
\hline & 9.00 & 74.2 & 83.2 & 4.40 (3.10 to 6.30$)$ \\
\hline & 10.00 & 59.7 & 91.0 & $6.64(4.00$ to 11.10$)$ \\
\hline \multirow[t]{3}{*}{$W P I C S_{\text {physical }}$} & 8.60 & 82.3 & 71.9 & 2.93 (2.30 to 3.80$)$ \\
\hline & 9.27 & 80.7 & 80.3 & 4.10 (3.00 to 5.70$)$ \\
\hline & 9.96 & 74.2 & 84.8 & 4.89 (3.40 to 7.10$)$ \\
\hline \multirow[t]{3}{*}{ PICS mental } & 6.00 & 91.9 & 68.5 & 2.92 (2.30 to 3.70$)$ \\
\hline & 7.00 & 82.3 & 78.1 & 3.75 (2.80 to 5.10$)$ \\
\hline & 8.00 & 77.4 & 81.5 & 4.18 (3.00 to 5.80$)$ \\
\hline \multirow[t]{3}{*}{$W P I C S_{\text {mental }}$} & 7.23 & 88.7 & 75.3 & 3.59 (2.70 to 4.70$)$ \\
\hline & 8.42 & 80.7 & 80.3 & $4.10(3.00$ to 5.70$)$ \\
\hline & 9.03 & 75.8 & 83.7 & 4.65 (3.20 to 6.70$)$ \\
\hline
\end{tabular}

$\mathrm{AUC}=$ Area under the curve; $\mathrm{Cl}=$ Confidence interval; $\mathrm{LR}=$ Likelihood ratio; $\mathrm{PICS}=$ Post-intensive care syndrome; $w \mathrm{PICS}=$ weighted PICS.

ference between AUCs of raw and weighted scores (Supplementary data).

\section{DISCUSSION}

We analysed AHP weights and cut-off values for each subscale and item to validate the PICSq, a measure of PICS in ICU survivors. The optimal cut-off values for screening unplanned readmissions of ICU survivors were 23.00 for the raw score and 23.73 for the weighted score, with AUCs of .933 and .929, respectively, corresponding to excellent accuracy [23]. There was no difference in accuracy between cut-off values for raw and weighted scores; thus, it may not be necessary to use weighted scores when assessing PICS. The AHP is a multi-criteria decision-making method in which experts assign weights through pairwise comparisons between items in each category $[17,26]$. Therefore, the weights in this study could be used by healthcare professionals to determine priorities of the needs or preferences of survivors.

The highest weight was assigned to the cognitive subscale of the PICSq. This can be interpreted that critical care experts perceive cognitive impairment as the most important factor of PICS. In addition, it is consistent with previous studies that reported that cognitive impairment experienced by critical care survivors had a higher incidence and longer duration than other areas of PICS [27-29]. The major risk factor for cognitive impairment is known as delirium [30], so ICU medical staff apply various interventions to prevent delirium. Recently, the use of ABCDEF Bundle has been recommended [31]. It has been reported that periodic assessment of consciousness and the use of appropriate analgesic and sedatives can reduce delirium [32].

Our expert AHP placed a high weight on items that differed from existing measurement tools commonly used (i.g., MoCA, HADS, measures of activities of daily living [ADL]). For example, the cognitive subscale item with the highest weight was "I am confused with date or time." This item could be more comprehensive and consistent than the items of the MoCA [8], which is recommended as a post-ICU cognitive evaluation tool, and gives 1 point each for orientation to the current year, month, and date. Most previous studies evaluated survivors' physical impairment using a 6-Minuite Walk Test (6MWT) or assessing ADL [7]. Unlike these, in our study, the item with the highest weight on the physical subscale was "I get tired easily," corresponding to a symptom survivors experience. The item with the highest weight on the mental subscale, "I have no hope," is also missing on the HADS, which experts recommend most to measure survivors' anxiety and depression [8]. The cause of these differences may be that the MoCA, ADL assessments, and HADS were not developed for ICU survivors. The weights in this study, along with the PICSq based on ICU survivors' experiences, can help to more appropriately assess survivors' cognitive, physical, and mental health.

Optimal cut-off values for measurement tools need to be determined by considering not only statistical accuracy but also clinical significance and usefulness [23]. In this study, sensitivity and specificity of 23.00 and 23.73 points, respectively, suggested optimal PICS ${ }_{\text {total }}$ and $w \mathrm{PICS}_{\text {total }}$ cut-off values were 80.9 85.5\%. If we had raised cut-off values, sensitivity could have been higher than 90\%; however, specificity would have been lowered to $70 \%$. 
We determined the optimal cut-off values by considering positive LR, which is the ratio of those who did and did not readmit among survivors above the cut-off. The high sensitivity of the PICSq can make it easier to identify survivors who are at-risk for re-admission, allowing for measures to be taken to reduce clinical and financial burdens [33]. However, we should also pay attention to the relatively low LR of our cut-off values. LR is the best indicator for ruling-in diagnoses, and the criteria for good diagnostic tests is LR >10 [23]. Compared to the sensitivity, LR is related to the usefulness of diagnostic tests [33]; thus, our findings suggested that the practical usefulness of the PICSq may be low. However, the PICSq is not a diagnostic test, rather it is a screening tool. It is a self-report measure that is free, non-invasive, and requires minimal time and effort. Therefore, low LR is unlikely to interfere with clinical use of the PICSq.

Healthcare providers can use our cut-off values to identify survivors likely to be readmitted and provide them with appropriate interventions. A quarter of our survivors reported unplanned readmissions at the time of the survey. We excluded planned hospitalization, such as chemotherapy or rehabilitation; therefore, readmission incidence appears lower than $70 \%$, as reported in a previous study [34]. Until 12 months post-discharge, ICU survivors' physical and mental health is at low levels and recovers slowly [35]. According to a relevant qualitative study [36], readmission breaks the trajectory of post-ICU recovery. Therefore, it is important to proactively assess unplanned readmission risk and provide appropriate interventions.

One intervention for ICU survivors is post-ICU clinics [27,37]. In post-ICU clinics, the 6MWT, MoCA, HADS, and assessment of ADL have been used for screenings and follow-up evaluations of survivors [38]. Using the PICSq cut-off values instead of these measures, which require space and assessor training, will increase the effectiveness of screenings, evaluations of interventions, and follow-up management. Specifically, using the cut-off values, nurses can easily screen PICS risk and help provide timely interventions to survivors in need. In addition, cut-off values can be used to evaluate various interventions such as therapeutic communication, family support, and the ICU diary provided for PICS prevention or reduction [39]. Because unplanned hospital readmission is a major long-term outcome indicator of critical care [15,40], our cut-off values could also contribute to the eval- uation and improvement of medical treatment and nursing care in the ICU. Additionally, PICS affects cognitive, physical, and mental health, and survivors may experience impairments in some or all areas [29]. We proposed the cut-off values for each subscale, as well as the PICSq total score, to provide a basis for more individualized assessments and interventions for PICS.

This study is meaningful in that it increased the usability of the PICSq by presenting cut-off values for the risk of unplanned readmission of ICU survivors. We proposed more valid cut-off values for screening hospital readmissions, for which various prevalence rates have been reported for different populations, by considering positive LR, as well as sensitivity and specificity. It is expected that our cut-off values for raw and weighted scores can be used for PICS screenings and intervention evaluations.

This study has some limitations. First, because participants were recruited voluntarily, survivors in relatively poor health would have been excluded. Thus, it is possible that overall PICS and readmission rates were underestimated. Second, participants self-reported their planned/unplanned hospital readmissions, and the accuracy of the reports cannot be guaranteed. In future studies, it is necessary to evaluate readmission by objective methods such as reviewing the participant's medical record. In addition, it is necessary to re-analyze the accuracy of the cut-off values by conducting a prospective study from the time the patient transferred from the ICU to the general inpatient unit. Third, this study was conducted at four university hospitals in three cities in Korea and did not include survivors who could not be discharged from the hospital after the ICU treatment or who had been transferred to other hospitals. Therefore, there may be limitations on the generalization of study results. As measurement accuracy is population dependent [23], repeated studies with different post-intensive care populations and settings are needed.

\section{CONCLUSION}

We calculated the weights of PICSq subscales and items through an expert group AHP, and suggested cut-off values for raw and weighted scores for unplanned readmission risk. The optimal cut-off values considering sensitivity, specificity, and positive LR were 23.00 for the raw score and 23.73 for the weighted score, with AUCs of .933 and .929, respectively, corre- 
sponding to excellent accuracy. Based on these findings, we recommend healthcare providers use the PICSq to screen for the risk of unplanned readmission and evaluate relevant interventions for ICU survivors.

\section{CONFLICTS OF INTEREST}

The authors declared no conflict of interest.

\section{ACKNOWLEDGEMENTS}

None.

\section{FUNDING}

This research was supported by Basic Science Research Program through the National Research Foundation of Korea (NRF) funded by the Ministry of Education (NRF-2016R1D1A1B0393 6044) and Korea government (MSIT) (No. NRF-2019R1A2C101 1300).

\section{DATA SHARING STATEMENT}

Please contact the corresponding author for data availability.

\section{AUTHOR CONTRIBUTIONS}

Conceptualization or/and Methodology: Kang J.

Data curation or/and Analysis: Hong J.

Funding acquisition: Kang J.

Investigation: Jeong YJ.

Project administration or/and Supervision: Kang J.

Resources or/and Software: Hong J.

Validation: Kang J \& Jeong YJ \& Hong J.

Visualization: Hong J.

Writing original draft or/and Review \& editing: Kang J \& Jeong YJ \& Hong J.

\section{SUPPLEMENTARY DATA}

Supplementary data to this article can be found online at https://doi.org/10.4040/jkan.20233.

\section{REFERENCES}

1. Kress JP, Hall JB. ICU-acquired weakness and recovery from critical illness. New England Journal of Medicine. 2014;370(17):16261635. https://doi.org/10.1056/NEJMra1209390

2. Iwashyna TJ. Trajectories of recovery and dysfunction after acute illness, with implications for clinical trial design. American Journal of Respiratory and Critical Care Medicine. 2012;186(4):302-304. https://doi.org/10.1164/rccm.201206-1138ED

3. Needham DM, Davidson J, Cohen H, Hopkins RO, Weinert $\mathrm{C}$, Wunsch $\mathrm{H}$, et al. Improving long-term outcomes after discharge from intensive care unit: Report from a stakeholders' conference. Critical Care Medicine. 2012;40(2):502-509. https://doi.org/10.1097/CCM.0b013e318232da75

4. Mikkelsen ME, Netzer G, Iwashyna T. Post-intensive care syndrome (PICS) [Internet]. Waltham (MA): UpToDate, Inc; c2019 [cited 2020 Nov 16]. Available from: http://www.uptodate.com/contents/post-intensive-care-syndrome-pics.

5. Blackwood B, Clarke M, McAuley DF, McGuigan PJ, Marshall JC, Rose L. How outcomes are defined in clinical trials of mechanically ventilated adults and children. American Journal of Respiratory and Critical Care Medicine. 2014;189(8):886893. https://doi.org/10.1164/rccm.201309-1645PP

6. Needham DM. Understanding and improving clinical trial outcome measures in acute respiratory failure. American Journal of Respiratory and Critical Care Medicine. 2014;189(8) :875877. https://doi.org/10.1164/rccm.201402-0362ED

7. Turnbull AE, Rabiee A, Davis WE, Nasser MF, Venna VR, Lolitha R, et al. Outcome measurement in ICU survivorship research from 1970 to 2013: A scoping review of 425 publications. Critical Care Medicine. 2016;44(7):1267-1277. https://doi.org/10.1097/CCM.0000000000001651

8. Needham DM, Sepulveda KA, Dinglas VD, Chessare CM, Friedman LA, Bingham CO 3rd, et al. Core outcome measures for clinical research in acute respiratory failure survivors. An international modified Delphi consensus study. American Journal of Respiratory and Critical Care Medicine. 2017;196(9):1122-1130. https://doi.org/10.1164/rccm.201702-03720C

9. Jeong YJ, Kang J. Development and validation of a questionnaire to measure post-intensive care syndrome. Intensive and Critical Care Nursing. 2019;55:102756. https://doi.org/10.1016/j.iccn.2019.102756

10. Kosilek RP, Baumeister SE, Ittermann T, Gründling M, Brunkhorst FM, Felix SB, et al. The association of intensive care with utilization and costs of outpatient healthcare services and quality of life. PloS One. 2019;14(9):e0222671. 
https://doi.org/10.1371/journal.pone.0222671

11. Lone NI, Gillies MA, Haddow C, Dobbie R, Rowan KM, Wild $\mathrm{SH}$, et al. Five-year mortality and hospital costs associated with surviving intensive care. American Journal of Respiratory and Critical Care Medicine. 2016;194(2):198-208. https://doi.org/10.1164/rccm.201511-22340C

12. Fischer C, Lingsma HF, Marang-van de Mheen PJ, Kringos DS, Klazinga NS, Steyerberg EW. Is the readmission rate a valid quality indicator? A review of the evidence. PloS One. 2014;9(11):e112282.

https://doi.org/10.1371/journal.pone.0112282

13. Lone NI, Seretny M, Wild SH, Rowan KM, Murray GD, Walsh TS. Surviving intensive care: A systematic review of healthcare resource use after hospital discharge. Critical Care Medicine. 2013;41(8) :1832-1843. https://doi.org/10.1097/CCM.0b013e31828a409c

14. Donaghy E, Salisbury L, Lone NI, Lee R, Ramsey P, Rattray $\mathrm{JE}$, et al. Unplanned early hospital readmission among critical care survivors: A mixed methods study of patients and carers. BMJ Quality \& Safety. 2018;27(11):915-927. https://doi.org/10.1136/bmjqs-2017-007513

15. Brown SM, Bose S, Banner-Goodspeed V, Beesley SJ, Dinglas VD, Hopkins RO, et al. Approaches to addressing post-intensive care syndrome among intensive care unit survivors. A narrative review. Annals of the American Thoracic Society. 2019;16(8):947-956. https://doi.org/10.1513/AnnalsATS.201812-913FR

16. Koksalmis E, Kabak Ö. Deriving decision makers' weights in group decision making: An overview of objective methods. Information Fusion. 2019;49:146-160. https://doi.org/10.1016/j.inffus.2018.11.009

17. Schmidt K, Aumann I, Hollander I, Damm K, von der Schulenburg JM. Applying the Analytic Hierarchy Process in healthcare research: A systematic literature review and evaluation of reporting. BMC Medical Informatics and Decision Making. 2015;15:112. https://doi.org/10.1186/s12911-015-0234-7

18. Saaty TL. Decision making with the analytic hierarchy process. International Journal of Services Sciences. 2008;1(1):83-98.

19. Waris M, Panigrahi S, Mengal A, Soomro MI, Mirjat NH, Ullah $\mathrm{M}$, et al. An application of analytic hierarchy process (AHP) for sustainable procurement of construction equipment: Multicriteria-based decision framework for Malaysia. Mathematical Problems in Engineering. 2019;2019:6391431. https://doi.org/10.1155/2019/6391431

20. Chen L, Chan CM, Lee HC, Chung Y, Lai F. Development of a decision support engine to assist patients with hospital selection. Journal of Medical Systems. 2014;38(6):59. https://doi.org/10.1007/s10916-014-0059-4

21. Dolan JG. Shared decision-making--transferring research into practice: The Analytic Hierarchy Process (AHP). Patient Ed- ucation and Counseling. 2008;73(3):418-425.

https://doi.org/10.1016/j.pec.2008.07.032

22. Song SW. Using the receiver operating characteristic (ROC) curve to measure sensitivity and specificity. Korean Journal of Family Medicine. 2009;30(11):841-842. https://doi.org/10.4082/kjfm.2009.30.11.841

23. Šimundić AM. Measures of diagnostic accuracy: Basic definitions. EJIFCC. 2009;19(4):203-211.

24. Fischer JE, Bachmann LM, Jaeschke R. A readers' guide to the interpretation of diagnostic test properties: Clinical example of sepsis. Intensive Care Medicine. 2003;29(7):10431051. https://doi.org/10.1007/s00134-003-1761-8

25. Dollaghan CA, Horner EA. Bilingual language assessment: A meta-analysis of diagnostic accuracy. Journal of Speech, Language, and Hearing Research. 2011;54(4):1077-1088. https://doi.org/10.1044/1092-4388(2010/10-0093)

26. Leal JE. AHP-express: A simplified version of the analytical hierarchy process method. MethodsX. 2020;7:100748. https://doi.org/10.1016/j.mex.2019.11.021

27. Harvey MA, Davidson JE. Postintensive care syndrome: Right care, right now... and later. Critical Care Medicine. 2016;44(2):381-385. https://doi.org/10.1097/CCM.0000000000001531

28. Iwashyna TJ, Ely EW, Smith DM, Langa KM. Long-term cognitive impairment and functional disability among survivors of severe sepsis. JAMA. 2010;304(16):1787-1794. https://doi.org/10.1001/jama.2010.1553

29. Marra A, Pandharipande PP, Girard TD, Patel MB, Hughes CG, Jackson JC, et al. Co-occurrence of post-intensive care syndrome problems among 406 survivors of critical illness. Critical Care Medicine. 2018;46(9):1393-1401. https://doi.org/10.1097/CCM.0000000000003218

30. Schulte PJ, Warner DO, Martin DP, Deljou A, Mielke MM, Knopman DS, et al. Association between critical care admissions and cognitive trajectories in older adults. Critical Care Medicine. 2019;47(8):1116-1124. https://doi.org/10.1097/CCM.0000000000003829

31. Marra A, Ely EW, Pandharipande PP, Patel MB. The ABCDEF bundle in critical care. Critical Care Clinics. 2017;33(2) :225243. https://doi.org/10.1016/j.ccc.2016.12.005

32. Devlin JW, Skrobik Y, Gélinas C, Needham DM, Slooter AJC, Pandharipande PP, et al. Clinical practice guidelines for the prevention and management of pain, agitation/sedation, delirium, immobility, and sleep disruption in adult patients in the ICU. Critical Care Medicine. 2018;46(9):e825-e873. https://doi.org/10.1097/CCM.0000000000003299

33. Trevethan R. Sensitivity, specificity, and predictive values: Foundations, pliabilities, and pitfalls in research and practice. Frontiers in Public Health. 2017;5:307. https://doi.org/10.3389/fpubh.2017.00307 
34. Williams TA, Knuiman MW, Finn JC, Ho KM, Dobb GJ, Webb SA. Effect of an episode of critical illness on subsequent hospitalisation: A linked data study. Anaesthesia. 2010;65(2):172177. https://doi.org/10.1111/j.1365-2044.2009.06206.x

35. Fan E, Dowdy DW, Colantuoni E, Mendez-Tellez PA, Sevransky JE, Shanholtz C, et al. Physical complications in acute lung injury survivors: A two-year longitudinal prospective study. Critical Care Medicine. 2014;42(4):849-859. https://doi.org/10.1097/CCM.0000000000000040

36. Kang J, Jeong YJ. Embracing the new vulnerable self: A grounded theory approach on critical care survivors' post-intensive care syndrome. Intensive and Critical Care Nursing. 2018;49:44-50. https://doi.org/10.1016/j.iccn.2018.08.004

37. Lasiter S, Oles SK, Mundell J, London S, Khan B. Critical care follow-up clinics: A scoping review of interventions and outcomes. Clinical Nurse Specialist CNS. 2016;30(4):227-
237. https://doi.org/10.1097/NUR.0000000000000219

38. Sevin CM, Bloom SL, Jackson JC, Wang L, Ely EW, Stollings JL. Comprehensive care of ICU survivors: Development and implementation of an ICU recovery center. Journal of Critical Care. 2018;46:141-148.

https://doi.org/10.1016/j.jcrc.2018.02.011

39. Inoue S, Hatakeyama J, Kondo Y, Hifumi T, Sakuramoto H, Kawasaki T, et al. Post-intensive care syndrome: Its pathophysiology, prevention, and future directions. Acute Medicine \& Surgery. 2019;6(3):233-246.

https://doi.org/10.1002/ams2.415

40. Hua M, Gong MN, Brady J, Wunsch H. Early and late unplanned rehospitalizations for survivors of critical illness. Critical Care Medicine. 2015;43(2):430-438. https://doi.org/10.1097/CCM.0000000000000717 